TRANSACTIONS OF THE

AMERICAN MATHEMATICAL SOCIETY

Volume 254, October 1979

\title{
ANALOGS OF CLIFFORD'S THEOREM FOR POLYCYCLIC-BY-FINITE GROUPS
}

BY

\author{
MARTIN LORENZ
}

\begin{abstract}
Let $P$ be a primitive ideal in the group algebra $K[G]$ of the polycyclic group $G$ and let $N$ be a normal subgroup of $G$. We show that among the irreducible right $K[G]$-modules with annihilator $P$ there exists at least one, $V$, such that the restricted $K[N]$-module $V_{N}$ is completely reducible, a sum of $G$-conjugate simple $K[N]$-submodules. Various stronger versions of this result are obtained. We also consider the action of $G$ on the factor $K[N] / P \cap K[N]$ and show that, in case $K$ is uncountable, any ideal $I$ of $K[N]$ satisfying $\bigcap_{g \in G} I^{8}=P \cap K[N]$ is contained in a primitive ideal $Q$ of $K[N]$ with $\bigcap_{g \in G} Q^{g}=P \cap K[N]$.
\end{abstract}

Introduction. By the classical restriction theorem of Clifford, we know that, given a finite group $G$ and an irreducible right $K[G]$-module $V$, then $V$ restricted to any normal subgroup $N$ of $G$ is a completely reducible $K[N]-$ module and its simple components are conjugate under $G$. In the case of infinite groups, this result is still true provided the normal subgroup $N$ has finite index in $G$ (cf. [15, Theorem 7.2.16]), but it fails to hold in general without this restriction on $N$. So consider, for instance, the two-generator nilpotent group of class two, $G=\langle x, y| z=[x, y]$ is central $\rangle$. If $K$ is a field containing an element $\lambda \in K^{\cdot}$ of infinite multiplicative order, then the Laurent polynomial ring $V=K\left[X^{ \pm 1}\right]$ becomes an irreducible right $K[G]$-module by the rules $X^{i} \cdot x=X^{i+1}, X^{i} \cdot y=\lambda^{i} X^{i}, X^{i} \cdot z=\lambda X^{i}$. But for the normal subgroup $N=\langle x, z\rangle$ of $G$, the restricted module $V_{N}$ has no irreducible $K[N]$-submodule, because such a submodule would correspond to a minimal ideal in $K\left[X^{ \pm 1}\right]$.

However, there is still something that can be said for an arbitrary normal subgroup $N$ of the polycyclic-by-finite group $G$. Namely, let $P$ be a primitive ideal in $K[G]$, say $P$ is the annihilator of the irreducible right $K[G]$-module $V$. In case $V_{N}$ splits into a sum of $G$-conjugate simple $K[N]$-submodules, an immediate consequence would be that

$$
P \cap K[N]=\bigcap_{g \in G} Q^{g}
$$

Received by the editors June 23, 1978.

AMS (MOS) subject classifications (1970). Primary 16A26, 20C05; Secondary 16A20.

Key words and phrases. Group ring, irreducible module, primitive ideal, maximal ideal, Ore extension, group acting on a group algebra, classical ring of quotients, Jacobson topology. 
for some primitive ideal $Q$ of $K[N]$. Now, it has been shown in [13] that such a primitive ideal $Q$ always exists, without any assumption on the primitive ideal $P$ and on the normal subgroup $N$. The present paper is an attempt to derive some module theoretic consequences of this fact.

In the first section, we show that, given a primitive ideal $P$ in the group algebra $K[G]$ of the polycyclic-by-finite group $G$ and given a normal subgroup $N$ of $G$, then there is at least one irreducible right $K[G]$-module $V$ with annihilator $P$ such that the restricted module $V_{N}$ is completely reducible. In fact, we prove slightly more (Theorem 1.6): Let $P$ be a primitive ideal of $K[G]$ and let $N$ be a normal subgroup of $G$. Then there exists a primitive ideal $Q$ of $K[N]$ such that

(i) $\cap_{g \in G} Q^{g}=P \cap K[N]$ and

(ii) for any irreducible $K[N]$-module $W$ with annihilator $Q$ there exists an irreducible $K[G]$-module $V$ with annihilator the given primitive ideal $P$ such that $V_{N}$ contains a copy of $W$. In particular, $V_{N}$ is isomorphic to a direct sum of $G$-conjugates of $W$. In the proof, we use the result of [13] mentioned above and extend it by Ore extension methods, that is by viewing $K[G]$ as essentially built up successively by ring extensions of the type $R \subset S$, where $S=R_{\alpha}[t]$ is an Ore extension.

The second section contains a sharpened version of the above result under the assumption that $P$ is a certain nicely behaved maximal ideal of $K[G]$. Without going into the details here, let us just mention that the conditions we impose on $P$ are satisfied by any primitive ideal in $K[G]$, if $G$ is finitely generated nilpotent. Roughly speaking, we prove in Corollary 2.5 , that for such a $P$ one may take any primitive ideal $Q$ of $K[N]$ satisfying the condition $\cap_{g \in G} Q^{g}=P \cap K[N]$ and one still has the module theoretic result as stated in part (ii) of the above Theorem 1.6.

In the remaining two sections, we are concerned with studying the action of the polycyclic-by-finite group $G$ on $R=K[N] / P \cap K[N]$, where $P$ is a primitive ideal in $K[G]$ and $N$ is a normal subgroup of $G$. In short, the action is such that there are "few" fixed elements in $R$ and "many" ideals in $R$ with a large orbit under $G$. More precisely, if $\mathcal{Q}(R)$ is the classical ring of quotients of $R$, then the fixed ring $2(R)^{G}$ under the obvious action of $G$ on $2(R)$ is finite dimensional over the ground field $K$. Furthermore, there are countably many nonzero $G$-stable ideals $I_{1}, I_{2}, \ldots$ of $R$ (not necessarily distinct and possibly equal to $R$ ) such that any ideal $I$ of $R$ with a small orbit under $G$, that is with $\cap_{g \in G} I^{g} \neq(0)$, must contain one of the ideals $I_{i}$. Here, the notions of small and large orbit are motivated by the so-called Jacobson topology on the set $\operatorname{Priv}(R)$ of primitive ideals of $R$. For the definition of this topology we refer to $\S 4$, and we just note here that the primitive ideal $Q$ of $R$ has a dense $G$-orbit in $\operatorname{Priv}(R)$ if and only if $\cap_{g \in G} Q^{g}=(0)$.

In terms of the Jacobson topology, the above result yields that, after 
removing at most countably many closed subsets from $\operatorname{Priv}(R)$, any primitive ideal of $R$ has a dense $G$-orbit. Of course, this does not say anything if the ground field $K$ itself is at most countable. But if $K$ is uncountable, then the result can be combined with a recent theorem of Farkas [4] to obtain among others the following theorem (Theorem 4.3): Let $K$ be an uncountable field, let $P$ be a primitive ideal of $K[G]$ and let $N$ be a polycyclic normal subgroup of $G$. Then any ideal $I$ of $R=K[N] / P \cap K[N]$ such that $\cap_{g \in G} I^{8}=(0)$ is contained in a primitive ideal $Q$ of $R$ such that $\cap_{g \in G} Q^{g}=(0)$. The methods of this last part of the paper are influenced by a recent article of Dixmier [3].

Our notation will be as in [15]. If not explicitly stated otherwise, $G$ will always be a polycyclic-by-finite group and $K$ will denote any commutative field. Rings are associative and have a 1, ideals are two-sided ideals and modules are understood to be unitary right modules.

1. Ore extension methods. In this section $R$ will always denote a right Noetherian $K$-algebra and $\alpha$ will be a $K$-algebra automorphism of $R$. The Ore extension $R_{\alpha}[t]$ is obtained from $R$ by adjoining an indeterminate $t$ subject to the equation

$$
\operatorname{tr}=r^{\alpha} t
$$

for all $r \in R$. Localizing $R_{\alpha}[t]$ at the powers of $t$, we obtain the skew Laurent polynomial ring $S=R_{\alpha}\left[t, t^{-1}\right]$. Thus a typical element of $S$ has the form

$$
f(t)=f_{m} t^{m}+f_{m+1} t^{m+1}+\cdots+f_{n} t^{n},
$$

where $f_{i} \in R$ and $m$ and $n$ are integers such that $m<n$. If $f_{m}$ and $f_{n}$ are nonzero, then we call $n-m$ the degree of $f(t)$. The automorphism $\alpha$ can be extended to an automorphism of $S$ via $t^{\alpha}=t$.

Suppose $\Gamma$ is a group acting as $K$-algebra automorphisms on $R$. Then $R$ is said to be $\Gamma$-prime if and only if the product of any two nonzero $\Gamma$-stable ideals of $R$ always is nonzero. A $\Gamma$-stable ideal $I$ of $R$ is called $\Gamma$-prime if $R / I$ is a $\Gamma$-prime ring. In the case $\Gamma=\langle\alpha\rangle$ we write $\alpha$-prime instead of $\langle\alpha\rangle$-prime. In the following lemma we gather some information from [7].

LeMmA 1.1. (i) If $R$ is $\Gamma$-prime, then $R$ is semiprime and the finitely many minimal prime ideals of $R$ from a complete orbit under $\Gamma$. Furthermore, any nonzero $\Gamma$-stable ideal of $R$ contains a regular element of $R$.

(ii) If $P$ is a prime ideal of $S=R_{\alpha}\left[t, t^{-1}\right]$, then $P \cap R$ is an $\alpha$-prime ideal of R.

(iii) Assume that the prime ideal $P$ of $S$ satisfies $P \neq(P \cap R) S$. If $I$ is an ideal of $S$ such that $I \supsetneqq P$, then $I \cap R \supsetneq P \cap R$.

Proof. The assertions are [7, Lemmas $1.5,1.3,1.10]$ or immediate consequences thereof.

Let $P$ denote a prime ideal of $R$. Then the heart of $P, \mathcal{H}(P)$, is defined to 
be the center of the simple Artinian classical ring of quotients of $R / P$, that is

$$
\mathscr{H}(P)=\mathscr{Z}(\mathcal{Q}(R / P))
$$

Thus the heart of a prime ideal is an extension field of $K$. If $R=K[G]$ is the group algebra of the polycyclic-by-finite group $G$, then the field extension $\mathcal{H}(P) / K$ is always finitely generated, by [13, Theorem 4.4$]$ or [12, Corollary 9]. Imitating terminology in [1] we make the following definition.

Definition. The prime ideal $P$ of the right Noetherian $K$-algebra $R$ is called almost rational if and only if the field extension $\mathcal{H}(P) / K$ is algebraic.

In the case $R=K[G]$, any primitive ideal $P$ of $R$ is almost rational. This follows from the fact that $\mathcal{H}(P)$ is embeddable in $\operatorname{End}_{R}(V)$ for any irreducible $R$-module $V$ with annihilator $P$, by [1, Satz 3.1], and this endomorphism ring is known to be algebraic over $K$ [9, Theorem 1.2].

LEMMA 1.2. Let $P$ denote an almost rational prime ideal of $S=R_{\alpha}\left[t, t^{-1}\right]$.

(i) If $P=(P \cap R) S$, then $\alpha^{l}$ in its action on $2(R / P \cap R)$ is an outer automorphism for all $l \neq 0$.

(ii) If $I$ is an ideal of $S$ such that $I \supsetneqq P$, then $I \cap R \supsetneq P \cap R$.

Proof. (i) Replacing $S$ by $S /(P \cap R) S \cong(R / P \cap R)_{\alpha}\left[t, t^{-1}\right]$ if necessary, we may assume that $P=(0)$. Let $\mathcal{L}=\mathcal{2}(R)$ and $\mathcal{L}=\mathcal{L}(S)$ denote the classical rings of quotients so that $2^{\prime} \subset \mathcal{2}$, and suppose some power $\alpha^{l}$, $l \neq 0$, acts as an inner automorphism on $2^{\prime}$. Then there exists a unit $q \in 2^{\prime}$ such that $q^{-1} r q=r^{\alpha^{\prime}}$ holds for all $r \in 2^{\prime}$. In particular, all $\alpha$-conjugates $q^{\alpha^{\prime}}$, $i \in \mathbf{Z}$, commute with each other and with $t^{l}$ and the elements $q^{\alpha^{\prime}} t^{l}=\left(q t^{l}\right)^{\alpha^{\prime}}$ $\in \mathcal{Q}$ centralize $\mathcal{Q}^{\prime}$. Hence

$$
y=q t^{l} q^{\alpha} t^{l} \cdot \ldots \cdot q^{\alpha^{\prime-1}} t^{l}=q q^{\alpha} \cdot \ldots \cdot q^{\alpha^{\prime-1}} t^{l^{2}}
$$

also centralizes $\mathcal{2}^{\prime}$. In addition, $y$ commutes with $t$ so $y$ is central in 2 . Since $y$ is clearly transcendental over $K$, we have a contradiction. Therefore, all powers $\alpha^{l}, l \neq 0$, act as outer automorphisms on $\mathcal{2}^{\prime}$.

(ii) If $P \neq(P \cap R) S$, then the result follows from Lemma 1.1(iii). Thus we have to consider the case $P=(P \cap R) S$. Again we may assume that $P=$ (0). In particular $R$ is $\alpha$-prime and semiprime, by Lemma 1.1(i) and (ii), and hence the regular elements of $R$ form a right Ore subset $\mathcal{C}$ of $R$ and of $S$ (see [7, Lemma 1.4]). We claim that the localization $S_{\mathcal{C}}$ is a simple ring. Since $S_{\mathcal{C}}$ can be identified with the skew Laurent polynomial ring $\mathscr{2}_{\alpha}^{\prime}\left[t, t^{-1}\right]$, where $\mathcal{2}^{\prime}=\mathcal{2}(R)$, it suffices to check that

(a) all powers $\alpha^{l}, l \neq 0$, act as outer automorphisms on $\mathcal{2}^{\prime}$ and

(b) $2^{\prime}$ does not contain an $\alpha$-stable ideal $\neq(0), \mathscr{2}^{\prime}$ (see, for instance, $[8$, Proposition 3]). But (a) is just assertion (i) and (b) follows from Lemma 1.1(i).

Now assume by way of contradiction that there exists an ideal $I \neq(0)$ of $S$ such that $I \cap R=(0)$. Choosing $I$ maximal with respect to these properties we may assume that $I$ is prime. But then, by [1, Satz 2.10], $I \mathcal{C}^{-1}=I S_{\mathcal{C}}$ is a 
nonzero prime ideal of $S_{\mathcal{C}}$ and so it contains the identity, by the above.

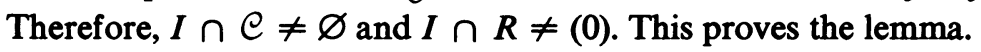

If $X$ is a right ideal of $R$, then we let $\operatorname{Id}(X)$ denote the greatest ideal of $R$ contained in $X$, that is $\operatorname{Id}(X)=\operatorname{ann}_{R}(R / X)$, the annihilator of the right $R$-module $R / X$.

LEMMA 1.3. If $X$ is a right ideal of $R$, then

$$
\operatorname{Id}(X S)=\left(\bigcap_{i \in \mathbf{Z}} \operatorname{Id}(X)^{\alpha^{i}}\right) \cdot S .
$$

Proof. The element

$$
f(t)=f_{m} t^{m}+f_{m+1} t^{m+1}+\cdots+f_{n} t^{n} \in S=R_{\alpha}\left[t, t^{-1}\right]
$$

is contained in $\operatorname{Id}(X S)$ if and only if for all $r \in R$ and any integer $i$ we have

$$
r t^{i} f(t)=r f_{m}^{\alpha^{i}} t^{m+i}+\cdots+r f_{n}^{\alpha^{i} t^{n+i} \in X S,}
$$

or equivalently, $r f_{l}^{\alpha^{i}} \in X$ for all $r \in R, i \in \mathbf{Z}$ and $l=m, m+1, \ldots, n$. Of course, the latter condition just says that $f_{l} \in \cap_{i \in \mathbf{Z}} \operatorname{Id}(X)^{\alpha^{\prime}}$ for all $l=m$, $m+1, \ldots, n$. The assertion follows.

The content of the following lemma is essentially a suitable application of the Euclidean algorithm to the skew Laurent polynomial ring $S=R_{\alpha}\left[t, t^{-1}\right]$. Part (i) will not be needed explicitly in the sequel but may be of some interest in its own right (see also Lemma 2.4).

LeMMA 1.4. Let $P$ be a prime ideal of $S=R_{\alpha}\left[t, t^{-1}\right]$.

(i) If $X$ is a right ideal of $R$ such that $\cap_{i \in \mathrm{Z}} \operatorname{Id}(X)^{\alpha^{t}}=P \cap R$, then $\operatorname{Id}(X S+P)=P$.

(ii) There exists an $\alpha$-stable ideal $I=I(P)$ of $R$ with $I^{n} \supset P \cap R$ for all $n$ and such that any right ideal $X$ of $R$ with $X \supset P \cap R$ and $X S+P=S$ contains some power $I^{n}$.

Proof. After factoring out the ideal $(P \cap R) S$ of $S$, if necessary, we may assume that $P \cap R=(0)$ and $R$ is $\alpha$-prime, the latter by Lemma 1.1(ii). If $P=(0)$, then

$$
\operatorname{Id}(X S+P)=\left(\bigcap_{i \in \mathbf{Z}} \operatorname{Id}(X)^{\alpha^{\prime}}\right) \cdot S=(0),
$$

by Lemma 1.3, and hence (i) holds. For (ii), we may choose $I=R$, for $X S=S$ implies $X=R$.

In the following we suppose that $P \neq(0)$. Choose an element

$$
f(t)=f_{m} t^{m}+f_{m+1} t^{m+1}+\cdots+f_{n} t^{n} \in P
$$

of minimal degree $n-m>0$. We may assume that $m=0$. Set $V=S / P$, $y=t+P \in V$. Then $R$ is a subring of the prime ring $V$ and is stable under conjugation with the unit $y \in V$. Let 


$$
A=\left\{r \in R \mid r y^{n} \in \sum_{i=0}^{n-1} R y^{i}\right\}
$$

and

$$
B=\left\{r \in R \mid r 1 \in \sum_{i=1}^{n} R y^{i}\right\} .
$$

Thus $\boldsymbol{A}$ is just the set of leading coefficients in $R$ of elements of degree $n$ in $P$, together with 0 , and $B$ is the set of lowest coefficients of elements of degree $n$ in $P$, together with 0 . It is easily checked that $A$ and $B$ both are nonzero $\alpha$-stable ideals of $R$. Inductively, one obtains that, for any nonnegative integer $l$,

$$
A^{l+1} y^{n+l}=y^{n+l} A^{l+1} \subset \sum_{i=0}^{n-1} R y^{i}
$$

and

$$
B^{l+1} y^{-l}=y^{-l} B^{l+1} \subset \sum_{i=1}^{n} R y^{i}
$$

Now let $I=A \cap B$. Then $I$ is an $\alpha$-stable ideal of $R$ and $I^{l} \neq(0)$ for all $l>0$, since $R$ is $\alpha$-prime. Furthermore, it follows from the above that for any integer $p$ there exists $l=l(p) \in \mathbf{Z}^{+}$such that

$$
y^{p} I^{l^{\prime}} \subset \sum_{i=0}^{n-1} R y^{i} \text { for all } l^{\prime}>l \text {. }
$$

Suppose $X$ is a right ideal of $R$ such that $\bigcap_{i \in \mathbf{Z}} \operatorname{Id}(X)^{\alpha^{\prime}}=(0)$ and assume by way of contradiction that $\operatorname{Id}(X S+P) \neq P$. Considered in $V$, this means the existence of a nonzero ideal $J$ of $V$ such that $J \subset X V$. By Lemma 1.1(iii), $J \cap R$ is a nonzero $\alpha$-stable ideal of $R$ which is contained in $X V=\Sigma_{i \in \mathbf{z}^{X}} X y^{i}$. Since $J \cap R$ is finitely generated as a right ideal of $R$, we may even choose integers $p$ and $q$ such that

$$
J \cap R \subset \sum_{i=p}^{q} X y^{i}
$$

There exists an integer $l$ with

$$
\left(\sum_{i=p}^{q} X y^{i}\right) I^{l} \subset \sum_{i=0}^{n-1} X y^{i}
$$

(for instance

$$
l=\max \{l(p), l(p+1), \ldots, l(q)\}
$$

works) and hence

$$
(J \cap R) I^{l} \subset \sum_{i=0}^{n-1} X y^{i}
$$


By the minimality of $n$, this implies that $(J \cap R) I^{l} \subset X$. Furthermore, the ideal $(J \cap R) I^{l}$ of $R$ is $\alpha$-stable and the $\alpha$-primeness of $R$ implies that it is nonzero. Therefore, we finally obtain the contradiction

$$
(0) \neq(J \cap R) I^{l} \subset \bigcap_{i \in \mathbf{Z}} \operatorname{Id}(X)^{\alpha^{\prime}}=(0) .
$$

Thus we must have $\operatorname{Id}(X S+P)=P$, and (i) is proved.

As to (ii), we choose $I$ to be the ideal of $R$ constructed above. If $X$ is a right ideal of $R$ such that $X S+P=S$, then $X V=V$ and there exist integers $p$ and $q$ such that $1 \in \sum_{i=p}^{q} X y^{i}$. Again, we use the fact that for a suitable power $I^{l}$ we have

$$
\left(\sum_{i=p}^{q} X y^{i}\right) I^{l} \subset \sum_{i=0}^{n-1} X y^{i}
$$

and hence $I^{l} \subset \sum_{i=0}^{n-1} X y^{i}$. The minimality of $n$ again implies that $I^{l} \subset X$, and we are done.

Before we apply this to group algebras, let us quote the following finite index lemma. Here and in the remainder of this section $G$ will always denote a polycyclic-by-finite group.

LEMMA 1.5. Let $N$ be a normal subgroup of finite index in $G$.

(i) If $P$ is a prime ideal in $K[G]$, then $P$ is minimal over $(P \cap K[N]) K[G]$.

(ii) Let $P$ be a primitive ideal of $K[G]$ and let $Q$ be a minimal covering prime of $P \cap K[N]$. If $V$ is an irreducible $K[G]$-module with annihilator $P$, then the restricted module $V_{N}$ contains an irreducible submodule $W$ with annihilator $Q$. In particular, $Q$ is primitive.

(iii) Let $Q$ be a primitive ideal of $K[N]$ and let $P$ be a minimal covering prime of $\left(\cap_{g \in G} Q^{g}\right) K[G]$. If $W$ is an irreducible $K[N]$-module with annihilator $Q$, then the induced module $W^{G}=W \otimes_{K[N]} K[G]$ has a composition factor $V$ such that $\operatorname{ann}_{K[G]}(V)=P$ and $V_{N} \supseteq W$. In particular, $P$ is primitive.

Proof. Assertion (i) is [13, Lemma 4.3(i)] and (ii), (iii) are proved in [13, Lemma 5.1].

We now come to the main result of this section.

TheOREM 1.6. Let $P$ be a primitive ideal of $K[G]$ and let $N$ be a normal subgroup of $G$. Then there exists a primitive ideal $Q$ of $K[N]$ such that:

(i) $\cap_{g \in G} Q^{g}=P \cap K[N]$;

(ii) for each irreducible $K[N]$-module $W$ with annihilator $Q$ there exists an irreducible $K[G]$-module $V$ with annihilator $P$ such that $V_{N} \supseteq W$. In particular, $V_{N}$ is isomorphic to a direct sum of $G$-conjugates of $W$.

Proof. Suppose $U$ is a normal subgroup of $G$ with $N \subset U$ and assume the assertion of the theorem holds for the pairs $(N, U)$ and $(U, G)$. Then it is 
easily checked that the theorem also holds for $(N, G)$. Since $G$ is polycyclic-by-finite, this remark reduces the problem to the following two cases:

$G / N$ is finite and $G / N$ is infinite cyclic. The case $G / N$ finite is contained in Lemma 1.5 , so we have to consider the case $G / N \cong \mathbf{Z}$ here, say $G=$ $\langle N, t\rangle$. Then $K[G] \cong K[N]_{\alpha}\left[t, t^{-1}\right]$, and we may apply our results on skew Laurent polynomial rings. First however, we infer from [13, Corollary 5.3] that there is a primitive ideal $Q$ of $K[N]$ such that $\cap_{g \in G} Q^{g}=P \cap K[N]$. Let $W$ be an irreducible $K[N]$-module with annihilator $Q$ and write $W \simeq$ $K[N] / X$ for some maximal right ideal $X$ of $K[N]$. We claim that $X K[G]+P$ is distinct from $K[G]$. Suppose otherwise and let $I=I(P)$ be the $G$-stable ideal of $K[N]$ constructed in Lemma 1.4. Then the assumption $X K[G]+P$ $=K[G]$ implies that $X \supset I^{n}$ for some positive integer $n$.

Since $Q=\operatorname{Id}(X)$ is the greatest ideal of $K[N]$ contained in $X$, it follows that $Q \supset I^{n}$ and hence $Q \supset I$. Therefore, since $I$ is $G$-stable, we obtain that

$$
I \subset \bigcap_{g \in G} Q^{g}=P \cap K[N]
$$

On the other hand, by construction, $I$ strictly contains $P \cap K[N]$. This contradiction shows that we must in fact have $X K[G]+P \neq K[G]$. In particular, we may choose a maximal right ideal $Y$ of $K[G]$ with $X K[G]+P$ $\subset Y$. Let $V$ be the irreducible $K[G]$-module $V=K[G] / Y$. Then

$$
V N \supseteq K[N] / Y \cap K[N]=K[N] / X \cong W
$$

and clearly, the annihilator $A$ of $V$ contains $P$. Suppose that $A \supsetneqq P$. Then, since $P$ is almost rational, we conclude by Lemma 1.2(ii) that $A \cap K[N] \supsetneq$ $P \cap K[N]$. But

$$
A \cap K[N]=\operatorname{ann}_{K[N]}(V) \subset \operatorname{ann}_{K[N]}(W)=Q
$$

and hence

$$
A \cap K[N] \subset \bigcap_{g \in G} Q^{g}=P \cap K[N]
$$

This contradiction shows that $V$ has annihilator precisely $P$, and the theorem is proved.

ReMARKS 1.7. (a) In the proof of Theorem 1.6 we have used [13, Corollary 5.3]. For finitely generated nilpotent-by-finite groups one can avoid this by using the fact that in this case all primitive ideals of $K[G]$ are maximal (cf. [13, Corollary 5.6]): Reduce to the case $G / N$ infinite cyclic as above and then argue as follows. Choose $I=I(P)$ according to Lemma 1.4(ii). Then $I$ is a $G$-stable ideal of $K[N]$ strictly containing $P \cap K[N]$. Since $K[N]$ is a Jacobson ring (cf. [9, Corollary 1.3]) and since $P \cap K[N]$ is a semiprime ideal of $K[N]$, we can find a primitive ideal $Q$ of $K[N]$ such that $Q \supset P \cap K[N]$ 
but $Q \nsupseteq I$. We claim that $\cap_{g \in G} Q^{g}=P \cap K[N]$. Otherwise, $\left(\cap_{g \in G} Q^{8}\right) K[G]+P$ would be a two-sided ideal of $K[G]$ strictly containing $P$. Consequently, $\left(\cap_{g \in G} Q^{g}\right) K[G]+P=K[G]$, and Lemma 1.4(ii) yields that $\cap_{g \in G} Q^{g} \supset I^{n}$ for some $n$. But this clearly implies that $Q \supset I$, contradicting the choice of $Q$. Therefore, we must have $\cap_{g \in G} Q^{g}=P \cap K[N]$ as claimed. The proof can now be completed as above.

(b) The proof of the theorem shows that, at least in the cases $G / N$ finite or infinite cyclic, we may take $Q$ to be any primitive ideal of $K[N]$ satisfying $\cap_{g \in G} Q^{g}=P \cap K[N]$ and still have the fact that to any irreducible $K[N]-$ module $W$ with annihilator $Q$ there exists an irreducible $K[G]$-module $V$ with annihilator $P$ such that $V_{N} \supseteq W$.

2. Maximal ideals. Throughout this section $G$ will denote a polycyclic-byfinite group. Let $I$ be an ideal of $K[G]$. Following [16] we set

$$
I^{\dagger}=\{g \in G \mid 1-g \in I\} \text {. }
$$

Thus $I^{\dagger}$ is the kernel of the natural homomorphism $G \rightarrow K[G] / I$ and hence is a normal subgroup of $G$. Let $\Delta_{I}$ be the subgroup of $G$ defined by

$$
\Delta_{I} / I^{\dagger}=\Delta\left(G / I^{\dagger}\right)
$$

the f.c. center of $G / I^{\dagger}$. Then $\Delta_{I}$ is normal in $G$ and, using the terminology of [15], we say the ideal $I$ is $\Delta_{I}$-controlled if

$$
I=\left(I \cap K\left[\Delta_{I}\right]\right) K[G]
$$

As to the relevance of these ideals we note that Roseblade has shown in [16, Theorems C.1 and C.2] that $G$ always contains a certain characteristic subgroup $G_{0}=\operatorname{nio}(G)$ of finite index such that every prime ideal $P$ of $K\left[G_{0}\right]$ is $\Delta_{P}$-controlled. Furthermore, it is not hard to show, using work in [16], that in the case of an arbitrary polycyclic-by-finite group $G$ at least every completely prime ideal $P$ of $K[G]$ is $\Delta_{P}$-controlled (i.e., those ideals $P$ of $K[G]$ for which $K[G] / P$ has no zero divisors).

Note that if the ideal $I$ is $\Delta_{I}$-controlled, then the factor $K[G] / I$ has the structure of a crossed product

$$
K[G] / I \simeq\left(K\left[\Delta_{I}\right] / I \cap K\left[\Delta_{I}\right]\right)_{\alpha}^{\gamma}\left[G / \Delta_{I}\right]
$$

We briefly recall that the crossed product $R_{\alpha}^{\gamma}[H]$ of the group $H$ over the ring $R$ contains for each $x \in H$ an element $\bar{x} \in R_{\alpha}^{\gamma}[H]$ and every element of $R_{\alpha}^{\gamma}[H]$ can be written uniquely as a finite sum $\Sigma_{x \in H^{\prime}} r_{x} \bar{x}$ with $r_{x} \in R$.

The addition in $R_{\alpha}^{\gamma}[H]$ is defined componentwise and the multiplication is given by the formulas

$$
\bar{x} \bar{y}=\gamma(x, y) \overline{x y}, \quad r \bar{x}=\bar{x} r^{\alpha(x)}
$$

for all $x, y \in H$ and $r \in R$. Here $\alpha: H \rightarrow \operatorname{Aut}(R)$ and $\gamma: H \times H \rightarrow U(R)$, the group of units of $R$, are maps that have to satisfy certain technical conditions 
which make the multiplication in $R_{\alpha}^{\gamma}[H]$ associative. For details and elementary properties of crossed products we refer to [10]. Here we just note that in order to view $K[G] / I$ as a crossed product of $H=G / \Delta_{I}$ over $R=$ $K\left[\Delta_{I}\right] / I \cap K\left[\Delta_{I}\right]$ one associates to each coset $x \in H$ the element $\bar{x}=g_{x}+$ $I \in K[G] / I$, where $g_{x} \in G$ is a fixed element of the coset $x$. The point is that $I=\left(I \cap K\left[\Delta_{I}\right]\right) K[G]$ implies that these elements $\bar{x}$, where $x$ runs over $H$, form a basis of $K[G] / I$ as a free left $R$-module. The map $\alpha$ arises from the action of $G$ on $R$ and $\gamma(x, y)$ is the element $d+I$ with $d=g_{x} g_{y} g_{x y}^{-1}$.

The next lemma deals with the following situation. Let $S \subset R$ be rings and let $\alpha: H \rightarrow \operatorname{Aut}(R)$ and $\gamma: H \times H \rightarrow U(R)$ be as above such that in addition $\operatorname{Im} \gamma \subset U(S)$ and $\alpha(G)$ transforms $S$ into itself. Then with the obvious mappings $\alpha^{\prime}$ and $\gamma^{\prime}$ we have a crossed product $S_{\alpha^{\prime}}^{\gamma^{\prime}}[H]$ which is a subring of $R_{\alpha}^{\gamma}[H]$.

LEMMA 2.1. Let $\mathcal{S}=S_{\alpha^{\prime}}^{\gamma^{\prime}}[H] \subset \Re=R_{\alpha}^{\gamma}[H]$ be crossed products as described above. If $\left.S\right|_{S} R$, that is if $S$ is a direct summand of $R$ considered as a left $S$-module, then $\left.\mathcal{S}\right|_{\delta} R$.

Proof. By assumption, there exists a left $S$-submodule $T$ of $R$ such that $R=S \oplus T$. Writing $\mathcal{T}$ for the set of elements $\Sigma_{x \in H} \bar{x} t_{x} \in R$ with $t_{x} \in T$, we see that $\mathcal{T}$ is stable under addition and left multiplication with elements of $\mathcal{S}$. To see the latter, let $s \in S, t \in T$ and $x, y \in H$. Then

$$
(\bar{x} s)(\bar{y} t)=\gamma(x, y) \overline{x y} s^{\alpha(y)} t=\overline{x y} \gamma(x, y)^{\alpha(x y)} s^{\alpha(y)} t,
$$

and since $\gamma(x, y)^{\alpha(x y)} s^{\alpha(y)} \in S$, we have $\gamma(x, y)^{\alpha(x y)_{s} \alpha(v)} t \in T$ and so $(\bar{x} s)(\bar{y} t)$ $\in \mathcal{T}$. Observe that each element of $R$ can also be written uniquely as a finite sum $\Sigma_{x \in H} \bar{x} r_{x}$, and the elements of $\mathcal{S}$ are just those with $r_{x} \in S$ for all $x$. So we have a direct sum decomposition of $\Re$ into left $\delta$-modules $R=\delta \oplus \mathcal{T}$. This establishes our claim.

The following lemma is essentially well known (see, for instance, the proof of [13, Lemma 4.5]).

LEMMA 2.2. Let $S \subset R$ be rings and assume that $R$ is Noetherian as right $S$-module. If $X \neq(0)$ is a right ideal of $R$ containing a regular element of $R$, then $X \cap S \neq(0)$.

Proof. Let $c$ be the regular element given by hypothesis. Then since $R$ is Noetherian as a right $S$-module, there is a positive integer $m$ with

$$
c^{m} \in S+c S+\cdots+c^{m-1} S .
$$

We obtain a monic equation of the form

$$
c^{m}+c^{m-1} s_{m-1}+\cdots+c s_{1}+s_{0}=0
$$

with $s_{i} \in S$. Choose such a monic equation of minimal degree $m>1$. Then, by the regularity of $c, s_{0}$ has to be nonzero and certainly $s_{0}$ is contained in $X$, 
since $c$ is an element of $X$. Thus $0 \neq s_{0} \in X \cap S$ and $X \cap S \neq(0)$.

The next lemma is very similar to Lemma 1.3 . We therefore leave the easy proof to the reader.

LEMMA 2.3. Let $N$ be a normal subgroup of $G$ and let $X$ be a right ideal of $K[N]$. Then

$$
\operatorname{Id}(X K[G])=\left(\bigcap_{g \in G} X^{g}\right) K[G] .
$$

In the proof of the next lemma we need the concept of a $G$-prime ring which was defined in the first section. We shall use the following version of Lemma 1.1(ii) (cf. also Lemma 3.2): If $P$ is a prime ideal of $K[G]$ and $N$ is a normal subgroup of $G$, then $P \cap K[N]$ is a $G$-prime ideal of $K[N]$.

LEMMA 2.4. Let $P$ be $\Delta_{P}$-controlled primitive ideal of $K[G]$ and let $N$ be a normal subgroup of $G$. If $X$ is a right ideal of $K[N]$ such that $\cap_{g \in G} X^{8}=$ $P \cap K[N]$, then

$$
\operatorname{Id}(X K[G]+P)=P .
$$

Proof. Set $U=N \Delta_{P}$ and $Y=X K[U]+(P \cap K[U])$. Then $U$ is a normal subgroup of $G$ and by assumption on $P$ we have

$$
P=(P \cap K[U]) K[G]
$$

and so

$$
Y K[G]=X K[G]+P \text {. }
$$

Hence

$$
\operatorname{Id}(X K[G]+P)=\operatorname{Id}(Y K[G])=\left(\bigcap_{g \in G} Y^{g}\right) K[G],
$$

by Lemma 2.3. The assertion is therefore equivalent to

$$
\bigcap_{g \in G} Y^{g}=P \cap K[U] \text {. }
$$

Set $\mathscr{R}=K[U] / P \cap K[U]$ and $\mathcal{S}=K[N] / P \cap K[N]$. Then, by the above remark, $\delta \subset \Re$ are $G$-prime rings. From $P=\left(P \cap K\left[\Delta_{P}\right]\right) K[G]$ it follows that

$$
P \cap K[U]=\left(P \cap K\left[\Delta_{P}\right]\right) K[U]
$$

and

$$
P \cap K[N]=\left(P \cap K\left[N \cap \Delta_{P}\right]\right) K[N] .
$$

Hence $R$ and $\mathcal{S}$ can be considered as crossed products

$$
\begin{aligned}
\Re & =\left(K\left[\Delta_{P}\right] / P \cap K\left[\Delta_{P}\right]\right)_{\alpha}^{\gamma}\left[U / \Delta_{P}\right], \\
\mathcal{S} & =\left(K\left[N \cap \Delta_{P}\right] / P \cap K\left[N \cap \Delta_{P}\right]\right)_{\alpha^{\prime}}^{\gamma^{\prime}}\left[N / N \cap \Delta_{P}\right] .
\end{aligned}
$$


Since $U / \Delta_{P} \cong N / N \cap \Delta_{P}$ we can choose the coset representatives for $\Delta_{P}$ in $U$ to be in $N$. Hence both $\Re$ and $\delta$ are crossed products over the group $H=N / N \cap \Delta_{P}$. Writing

$$
R=K\left[\Delta_{P}\right] / P \cap K\left[\Delta_{P}\right] \text { and } S=K\left[N \cap \Delta_{P}\right] / P \cap K\left[N \cap \Delta_{P}\right]
$$

we have $S \subset R$, and the point about $P$ being primitive is that this implies $R$ to be finite dimensional over $K$ (see [11, Lemma 2.4] or Lemma 3.5). In particular, $S$ is a semisimple Artinian ring and hence $\left.S\right|_{S} R$. Lemma 2.1 allows us to conclude that $\left.\delta\right|_{\delta} \Re$. Let $\bar{Y}$ be the image of $Y$ in $\Re$ and let $\bar{X}$ be the image of $X$ in $\delta$. Then $\bar{Y}=\bar{X} \Re$ and, since $\left.\delta\right|_{\delta} R$, we obtain that $\bar{Y} \cap \mathcal{S}=\bar{X}$. Now $G$ certainly acts on $\Re$ and $\mathcal{S}$, and our assumption on $X$ can be expressed as $\cap_{g \in G} \bar{X}^{g}=(0)$, whereas our assertion, as modified in the first paragraph of the proof, becomes $\cap_{g \in G} \bar{Y}^{g}=(0)$. Assume by way of contradiction that $\cap_{g \in G} \bar{Y}^{8}$ is nonzero. Then since $\mathcal{R}$ is a $G$-prime ring and $\cap_{g \in G} \bar{Y}^{g}$ is a $G$-stable two-sided ideal of $\Re$, we could use Lemma 1.1(i) to conclude that there is a regular element in $\cap_{g \in G} \bar{Y}^{g}$. But as we observed above, $R$ is finite dimensional over $K$ and so $\Re$ is finitely generated as a right $\mathcal{S}$-module and hence is Noetherian as a right $\mathcal{S}$-module. We can therefore apply Lemma 2.2 to obtain that

$$
\bigcap_{g \in G} \bar{Y}^{g} \cap \delta \neq(0) .
$$

But since $\delta$ is $G$-stable, we have

$$
\bigcap_{g \in G} \bar{Y}^{8} \cap \delta=\bigcap_{g \in G}(\bar{Y} \cap \delta)^{g}=\bigcap_{g \in G} \bar{X}^{g}=(0) .
$$

This contradiction shows that we have in fact $\cap_{g \in G} \bar{Y}^{g}=(0)$, as was to be shown.

What we have obtained so far is the following: Given a $\Delta_{P}$-controlled primitive ideal $P$ of $K[G]$, a normal subgroup $N$ of $G$ and a primitive ideal $Q$ of $K[N]$ such that $\cap_{g \in G} Q^{g}=P \cap K[N]$, then to any irreducible $K[N]$ module $W$ with annihilator $Q$ there exists a $K[G]$-module $V$ with annihilator $P$ such that $V_{N}$ is isomorphic to a direct sum of $G$-conjugates of $W$. Namely, we just write $W$ in the form $W \cong K[N] / X$ with some maximal right ideal $X$ of $K[N]$. Since $Q$ is the annihilator of $W$, we have $\cap_{n \in N} X^{n}=Q$ and hence

$$
\bigcap_{g \in G} X^{g}=\bigcap_{g \in G} Q^{g}=P \cap K[N] .
$$

Therefore, the lemma gives $\operatorname{Id}(X K[G]+P)=P$, that is $P$ is the annihilator of the $K[G]$-module $V=K[G] /(X K[G]+P)$. Clearly, $V \supseteq K[N] / X \cong W$ and $V=\Sigma_{g \in G}(K[N] / X) g$. Hence $V_{N}$ is isomorphic to a direct sum of $G$-conjugates of $W$. Of course, $V$ will not be irreducible in general, and we do not know whether or not $V$ must necessarily have a composition factor with 
annihilator precisely $P$. Such a composition factor, considered as a $K[N]-$ module would also be isomorphic to a sum of conjugates of $W$. One case at least is trivial, namely the case where $P$ is $\Delta_{P}$-controlled and maximal, for then any composition factor of $V$ has annihilator precisely $P$. So we may state the following

Corollary 2.5. Let $P$ be a $\Delta_{P}$-controlled maximal ideal of $K[G]$, let $N$ be a normal subgroup of $G$ and let $Q$ be any primitive ideal of $K[N]$ such that $\cap_{g \in G} Q^{g}=P \cap K[N]$.

Then to any irreducible $K[N]$-module $W$ with annihilator $Q$ there exists an irreducible $K[G]$-module $V$ with annihilator $P$ such that $V_{N}$ is isomorphic to a direct sum of $G$-conjugates of $W$.

3. Almost fixed point free group actions. Throughout this section $G$ will denote a polycyclic-by-finite group. Let $\Gamma$ be a group acting on $K[G]$. Recall from $\S 1$ that an ideal $P$ of $K[G]$ is said to be $\Gamma$-prime if $P$ is $\Gamma$-stable and if $A B \subset P$ for $\Gamma$-stable ideals $A$ and $B$ of $K[G]$ implies that $A \subset P$ or $B \subset P$. By Lemma 1.1(i), any $\Gamma$-prime ideal $P$ of $K[G]$ is semiprime and in fact can be uniquely expressed in the form $P=P_{1} \cap P_{2} \cap \ldots \cap P_{n}$, where the ideals $P_{i}$ of $K[G]$ are prime and form a complete orbit under the action of $\Gamma$.

In the following, $\operatorname{Int}(G)$ denotes the group of inner automorphisms of $G$, considered as a group of automorphisms on $K[G]$.

LEMMA 3.1. Let $\Gamma$ be a group acting on $K[G]$ and let $P$ be a $\Gamma$-prime ideal of $K[G]$. Then $\Gamma$ acts on the classical ring or quotients $2(K[G] / P)$ of $K[G] / P$. If $\operatorname{Int}(G) \subset \Gamma$, then the fixed ring $2(K[G] / P)^{\Gamma}$ is a finitely generated field extension of $K$.

Proof. Write $P=P_{1} \cap P_{2} \cap \ldots \cap P_{n}$, a finite intersection of $\Gamma$-conjugate prime ideals $P_{i}=P_{1}^{\gamma_{i}}$. By [6, Theorem 2.17], none of the ideals $P_{i}$ contains an element which is regular modulo $P$. Hence the right ideals $\bar{P}_{i} \mathcal{Q}$ of $\mathcal{Q}=$ $\mathcal{2}(K[G] / P)$ are actually two-sided and are precisely the prime ideals of 2 (cf. [1, Satz 2.10]). Here we have written $\bar{P}_{i}=P_{i} / P$. We obtain $K$-preserving isomorphisms

$$
\mathcal{Q} \cong \sum_{i=1}^{n} \oplus \mathcal{Q} / \bar{P}_{i} \mathcal{Q} \simeq\left(\mathcal{Q} / \bar{P}_{1} \mathcal{Q}\right)^{n}
$$

sending the element $q \in \mathcal{Q}$ to the $n$-tuple $\left(q^{\gamma_{i}^{-1}}+\bar{P}_{1} \mathcal{Q}\right)_{i}$ in $\left(\mathcal{Q} / \bar{P}_{1} \mathcal{Q}\right)^{n}$. Under this map, the elements of $\mathscr{Q}^{\Gamma}$ have their images in the diagonal of $\left(\mathcal{Q} / \bar{P}_{1} \mathcal{Q}\right)^{n}$ and, since $\operatorname{Int}(G) \subset \Gamma$, the components of the image of $q \in \mathcal{Q}^{\Gamma}$ are stable under the action of $G$ on $2 / \bar{P}_{1} \mathcal{Q}$. Therefore, we have a $K$-algebra embedding

$$
\mathcal{Q}^{\Gamma} \hookrightarrow \operatorname{diag}\left(\mathcal{Q} / \bar{P}_{1} \mathcal{Q}\right)^{G} \simeq \mathscr{Z}\left(\mathcal{Q} / \bar{P}_{1} \mathcal{Q}\right)
$$


Now $2 / \bar{P}_{1} \mathcal{2}$ is canonically isomorphic to the ring of quotients $2\left(K[G] / P_{1}\right)$ of $K[G] / P_{1}$ (see [1, Satz 2.10]) and hence $\mathscr{Z}\left(2 / \bar{P}_{1} 2\right)$ is the heart of $P_{1}$,

$$
\mathcal{H}\left(P_{1}\right)=\mathscr{Z}\left(\mathcal{2}\left(K[G] / P_{1}\right)\right)
$$

But the latter is known to be a finitely generated field extension of $K$, by [13, Theorem 4.4]. Therefore, the subextension $2^{\Gamma}$ is also finitely generated, and the lemma is proved.

Definition. Let $\Gamma$ be a group acting as $K$-algebra automorphisms on $K[G]$ such that $\operatorname{Int}(G) \subset \Gamma$. Let $P$ be a $\Gamma$-prime ideal of $K[G]$. We say that the action of $\Gamma$ on $K[G] / P$ is almost fixed point free if and only if the field extension $2(K[G] / P)^{\Gamma} / K$ is algebraic (and hence is finite dimensional).

To give a first example, let $\tau$ be an automorphism of the finitely generated torsion free abelian group $A$ such that 1 is the only element of $A$ with a finite orbit under $\tau$. In this case, the action of $\langle\tau\rangle$ on $K[A]$ is almost fixed point free in the sense of the above definition. For, the semidirect product $G=A\langle\tau\rangle$ has f.c. center $\Delta(G)=\langle 1\rangle$ (see [5, Lemma 4.4]), and this implies $\mathscr{Z}(\mathcal{2}(K[G]))=K$, by [15, Theorem 4.4.5]. But obviously, $\mathcal{Q}(K[A])^{\tau} \subset$ $\mathscr{Z}(\mathcal{2}(K[G]))$ and so $\mathcal{L}(K[A])^{\tau}=K$. The converse is also true and is in fact easier.

Our main interest in almost fixed point free group actions stems from the fact that, in case $P$ is a primitive ideal of $K[G]$, the action of $G$ on $K[G] / P$ is almost fixed point free. In fact, this is just an alternative way of expressing that primitive ideals in $K[G]$ are almost rational (cf. $\$ 1$ ). The following lemma and its corollary extend this observation to subrings $K[N] / P \cap$ $K[N]$, where $N$ is normal in $G$. Here and in the remainder of this section we are concerned with the following special situation: $\Gamma$ is a group containing $G$ as a normal subgroup and $\Gamma$ acts on $K[G]$ and suitable factors of $K[G]$ via its action on $G$.

LEMMA 3.2. Let $G \triangleleft \Gamma$ and let $P$ be $a \Gamma$-prime ideal of $K[G]$. If $N \subset G$ is normal in $\Gamma$, then $P \cap K[N]$ is a $\Gamma$-prime of $K[N]$ and we have a $K$-algebra embedding of the fixed rings

$$
\mathcal{2}(K[N] / P \cap K[N])^{\Gamma} \hookrightarrow \mathcal{Q}(K[G] / P)^{\Gamma} .
$$

In particular, if the action of $\Gamma$ on $K[G] / P$ is almost fixed point free, then the same holds for the subring $K[N] / P \cap K[N]$.

Proof. Clearly, $P \cap K[N]$ is $\Gamma$-stable. Let $A$ and $B$ be $\Gamma$-stable ideals of $K[N]$ such that $A B \subset P \cap K[N]$. Then $A$ and $B$ are in particular $G$-stable and so $A K[G]=K[G] A$ and $B K[G]=K[G] B$ are two-sided $\Gamma$-stable ideals of $K[G]$. Since

$$
(A K[G])(B K[G])=(A B) K[G] \subset(P \cap K[N]) K[G] \subset P,
$$

the assumption on $P$ implies that $A K[G] \subset P$ or $B K[G] \subset P$ and hence 
$A \subset P \cap K[N]$ or $B \subset P \cap K[N]$. This shows that $P \cap K[N]$ is, in fact, $\Gamma$-prime.

Set $R=K[G] / P$ and $S=K[N] / P \cap K[N]$. Then $S$ is a $\Gamma$-prime subring of $R$, and we have to show that $2(S)^{\Gamma}$ embeds in $2(R)^{\Gamma}$. So let $r$ and $s$ be elements of $S$, with $s$ regular in $S$, such that $z=r s^{-1} \in \mathcal{2}(S)^{\Gamma}$. Then in particular, $z$ is central in $\mathcal{Q}(S)$ and for all $\gamma \in \Gamma$ we have

$$
r^{\gamma_{S}}=(z s)^{\gamma} s=z^{\gamma_{s}{ }^{\gamma}} s=z s^{\gamma_{S}}=s^{\gamma_{z S}}=s^{\gamma} r .
$$

Interpreting the equation $r^{\gamma} s=s^{\gamma} r$ in $R$ we obtain that $r x s=s x r$, for all $x \in R$. Now let $P_{1}, P_{2}, \ldots, P_{n}$ denote the minimal primes of $R$ and set $r_{i}=r+P_{i}, s_{i}=s+P_{i} \in R / P_{i}$. Then for all $i$ and for all $x \in R / P_{i}, r_{i} x s_{i}=$ $s_{i} x r_{i}$. It follows from a result of Martindale [14, Theorem 4], that for each $i$ there exists $c_{i} \in \mathscr{Z}\left(\mathcal{L}\left(R / P_{i}\right)\right)$ with $r_{i}=c_{i} s_{i}$. As in the proof of Lemma 3.1, we identify $\mathcal{L}=2(R)$ with $\sum_{i=1}^{n} \oplus \mathcal{Q}_{i}$, where $\mathcal{Q}_{i}=\mathcal{2} / P_{i} \mathcal{Q}=\mathcal{2}\left(R / P_{i}\right)$. We have $r=r_{1}+r_{2}+\cdots+r_{n}, s=s_{1}+s_{2}+\cdots+s_{n}$ and $r=c s$, where $c=$ $c_{1}+c_{2}+\cdots+c_{n}$ is central in 2 . Therefore, in 2 the equation $r^{\gamma} s=s^{\gamma} r$ reads as $c^{\gamma}{ }^{\gamma} s=s^{\gamma} c s=c s^{\gamma} s$. Thus we have

$$
\left(c^{\gamma}-c\right) s^{\gamma} s=0
$$

for all $\gamma \in \Gamma$. Now let $\pi_{i}$ denote the projection of $\mathcal{Q}=\sum_{i=1}^{n} \oplus \mathcal{Q}_{i}$ onto the $i$ th component. Observe that no $\pi_{i}$ annihilates a regular element of $S$. For this would imply that $P_{i}$ contains a regular element of $S$ and hence each of the ideals $P_{1}, P_{2}, \ldots, P_{n}$ contains a regular element of $S$, since the $\Gamma$-primeness of $R$ implies that these ideals are conjugate under $\Gamma$. However, the product $P_{1} \cdot P_{2} \cdot \cdots \cdot P_{n}$ is zero, a contradiction. In particular, $\pi_{i}\left(s^{\gamma} s\right)$ is nonzero for all $\gamma$ and since $\pi_{i}\left(c^{\gamma}-c\right)$ is central in the prime ring $\mathcal{Q}_{i}$ for all $\gamma$, we deduce from $\pi_{i}\left(c^{\gamma}-c\right) \pi_{i}\left(s^{\gamma} s\right)=0$ that $\pi_{i}\left(c^{\gamma}-c\right)=0$ for $i=1,2, \ldots, n$ and for all $\gamma \in \Gamma$. This shows that $c$ is $\Gamma$-invariant and so we have obtained that $r=c s$, for some $c \in 2^{\Gamma}$. We show that $c$ only depends on $z$, not on the choices of $r$ and $s$. For, if $z$ is written in the form $z=(r u)(s u)^{-1}$ with $u$ regular in $S$, then as above we obtain $c^{\prime} \in \mathcal{Q}^{\Gamma}$ such that $r u=c^{\prime} s u$. On the other hand, $r u=c s u$ and hence $\left(c-c^{\prime}\right) s u=0$. Note since $R$ is $\Gamma$-prime so is 2. Thus if $c \neq c^{\prime}$, then $c-c^{\prime}$ is a regular element of 2 and $\left(c-c^{\prime}\right) s u=0$ implies that $s u=0$, a contradiction. Hence we see that $c=c^{\prime}$. Routine verification shows that the map $z \mapsto c$ from $2(S)^{\Gamma}$ to $\mathcal{2}^{\Gamma}=\mathcal{2}(R)^{\Gamma}$ is a $K$-algebra homomorphism which has to be an embedding, since $2(S)^{\Gamma}$ is a field (Lemma 3.1). The last assertion of the lemma is an immediate consequence of this embedding.

In view of the remarks made before the statement of the lemma we have the following:

Corollary 3.3. Let $P$ be a primitive ideal of $K[G]$. Then for any normal subgroup $N$ of $G$, the action of $G$ on $K[N] / P \cap K[N]$ is almost fixed point free. 
LEMMA 3.4. Let $G \triangleleft \Gamma$ and let $P$ be a $\Gamma$-prime ideal of $K[G]$.

(i) If $\Gamma_{0} \triangleleft \Gamma$, then $P$ can be uniquely expressed as a finite irredundant intersection $P=P_{1} \cap P_{2} \cap \ldots \cap P_{n}$ of $\Gamma_{0}$-prime ideals $P_{i}$ which form an orbit under the action of $\Gamma$.

(ii) Suppose the normal subgroup $\Gamma_{0}$ of $\Gamma$ has finite index and set $G_{0}=G \cap$ $\Gamma_{0}$. Then

$$
P \cap K\left[G_{0}\right]=\bigcap_{\gamma \in \Gamma} P_{0}^{\gamma},
$$

where $P_{0}$ is a $\Gamma_{0}$-prime of $K\left[G_{0}\right]$ which is uniquely determined up to $\Gamma$-conjugation. Furthermore, if the action of $\Gamma$ on $K[G] / P$ is almost fixed point free, then the same holds for the action of $\Gamma_{0}$ on $K\left[G_{0}\right] / P_{0}$.

Proof. Observe that $\Gamma$ permutes the minimal covering primes $Q_{1}, Q_{2}, \ldots, Q_{r}$ of $P$ transitively and $P$ equals their intersection. The action of $\Gamma_{0}$ on $\left\{Q_{1}, Q_{2}, \ldots, Q_{r}\right\}$ produces orbits $\theta_{1}, \theta_{2}, \ldots, \theta_{n}$ which again are permuted transitively by $\Gamma$, since $\Gamma_{0}$ is normal in $\Gamma$. Therefore, the ideals $P_{i}=\cap_{Q \in \mathcal{O}_{i}} Q$ form an orbit under $\Gamma$, they are $\Gamma_{0}$-prime and $P=\cap P_{i}$. The proof of the uniqueness is easy and is omitted.

It remains to prove (ii). First, by Lemma 3.2, $P \cap K\left[G_{0}\right]$ is $\Gamma$-prime and hence the existence and uniqueness (up to $\Gamma$-conjugacy) of an expression of the form

$$
P \cap K\left[G_{0}\right]=\bigcap_{\gamma \in \Gamma} P_{0}^{\gamma}
$$

with $P_{0}$ a $\Gamma_{0}$-prime follows from part (i). Suppose now that the action on $K[G] / P$ is almost fixed point free. We want to show that $2\left(K\left[G_{0}\right] / P_{0}\right)^{\Gamma_{0}}$ is finite dimensional over $K$.

From Lemma 3.2 we know that $2^{\Gamma}$ is finite dimensional, where we have written $2=2\left(K\left[G_{0}\right] / P \cap K\left[G_{0}\right]\right)$. Write $P \cap K\left[G_{0}\right]=P_{1} \cap P_{2}$ $\cap \ldots \cap P_{n}$, an irredundant intersection of $\Gamma_{0}$-prime ideals $P_{i}$ which are permuted transitively by $\Gamma$. In analogy with the proof of Lemma 3.1, we write $\mathcal{Q}$ as the sum

$$
\mathcal{Q}=\sum_{i=1}^{n} \oplus \mathcal{Q}\left(K\left[G_{0}\right] / P_{i}\right)
$$

The group $\Gamma$, in its action on 2 , permutes these summands transitively and $\Gamma_{0}$ stabilizes them. Therefore,

$$
2^{\Gamma}=\left(2^{\Gamma_{0}}\right)^{\Gamma / \Gamma_{0}}=\left(\sum_{i=1}^{n} \bigoplus \mathcal{Q}\left(K\left[G_{0}\right] / P_{i}\right)^{\Gamma_{0}}\right)^{\Gamma / \Gamma_{0}} .
$$

Set $\bar{\Gamma}=\Gamma / \Gamma_{0}$ and $F_{i}=\mathcal{2}\left(K\left[G_{0}\right] / P_{i}\right)^{\Gamma_{0}}$. Then $\bar{\Gamma}$ is a finite group and each $F_{i}$ is a finitely generated field extension of $K$, by Lemma 3.1, $F_{i} \simeq F_{1}$. Our claim can be expressed as $\left(F_{1}: K\right)<\infty$. Set 


$$
\overline{\Gamma_{1}}=\left\{x \in \bar{\Gamma} \mid F_{1}^{x}=F_{1}\right\} .
$$

Then the finite group $\overline{\Gamma_{1}}$ acts as field automorphisms on $F_{1}$ and fixes the elements of $K$. Hence, if $K_{1}$ denotes the fixed field of $\overline{\Gamma_{1}}$, then $K \subset K_{1}$ and $\left(F_{1}: K_{1}\right)<\infty$. So it suffices to show that $\left(K_{1}: K\right)<\infty$. Choose a right transversal $\left\{x_{1}, x_{2}, \ldots, x_{r}\right\}$ for $\bar{\Gamma}_{1}$ in $\bar{\Gamma}$. If $\lambda \in K_{1}$, then the element

$$
\lambda^{x_{1}}+\lambda^{x_{2}}+\cdots+\lambda^{x_{r}} \in 2
$$

is clearly independent of the choice of the transversal and is contained in $2^{\Gamma}$. Finally, the map

$$
\lambda \mapsto \lambda^{x_{1}}+\lambda^{x_{2}}+\cdots+\lambda^{x_{1}}
$$

is easily seen to be a one-to-one ring homomorphism. Thus, as a $K$-algebra $K_{1}$ is embedded isomorphically in $2^{\Gamma}$ and so $\left(K_{1}: K\right)<\left(2^{\Gamma}: K\right)<\infty$. This proves the lemma.

Lemma 3.5. Let $G \triangleleft \Gamma$ and let $P$ be a $\Gamma$-prime ideal of $K[G]$. Suppose that the action of $\Gamma$ on $K[G] / P$ is almost fixed point free and assume $G=D_{G}(\Gamma)=$ $\{x \in G \mid x$ has only finitely many $\Gamma$-conjugates $\}$. Then $K[G] / P$ is finite dimensional over $K$. In particular, $P$ is maximal among the $\Gamma$-stable ideals of $K[G]$.

Proof. Set $Z=\mathscr{Z}(G)$ and $R=K[Z] / P \cap K[Z]$. Then $Z$ has finite index in $G$ and is normal in $\Gamma$. Hence $\Gamma$ acts on $R$ and in fact some finite image of $\Gamma$ is acting, since $G=\mathbf{D}_{G}(\Gamma)$. We conclude that $R$ is integral over the fixed ring $R^{\Gamma}$ and $R$ is a finitely generated $R^{\Gamma}$-module (cf. [2, p. 33]). Since $R^{\Gamma} \subset$ $\mathcal{2}(K[G] / P)^{\Gamma}$, the assumption on $\Gamma$ implies that $R^{\Gamma}$ is finite dimensional over $K$. Hence $R$ is also finite dimensional, and

$$
\operatorname{dim}_{K} K[G] / P<\operatorname{dim}_{K} R \cdot[G: Z]<\infty .
$$

As to the final assertion, observe that by Lemma 1.1(i), $P$ can be written in the form $P=\cap_{\gamma \in \Gamma} Q^{\gamma}$, where $Q$ is a prime ideal of $K[G]$ and the intersection is finite. Since $P$ has finite codimension in $K[G]$, the ideal $Q$ is even maximal. Therefore, any ideal of $K[G]$ containing $P$ is contained in some $Q^{\gamma}$ and any $\Gamma$-stable ideal over $P$ is contained in $\cap_{\gamma \in \Gamma} Q^{\gamma}=P$.

Recall from [16] that a group $\Gamma$ is said to be orbitally sound if and only if any subgroup $U$ of $\Gamma$ having only finitely many $\Gamma$-conjugates satisfies $\left[U: \cap_{\gamma \in \Gamma} U^{\gamma}\right]<\infty$.

An ideal $I$ of $K[\Gamma]$ is said to be faithful, if $I^{\dagger}=\langle 1\rangle$, and almost faithful, if $I^{\dagger}$ is finite. The key results of [16] we need are as follows.

THEOREM 3.6 (RoSEBLADE). Let $\Gamma$ be polycyclic-by-finite.

(i) $\Gamma$ has a normal subgroup $\Gamma_{0}$ of finite index which is orbitally sound.

(ii) Let $\Gamma$ be orbitally sound and let $G \triangleleft \Gamma$. Set $F=\mathbf{D}_{G}(\Gamma)$. If $P$ is an almost faithful $\Gamma$-prime ideal of $K[G]$, then $P=(P \cap K[F]) K[G]$. 
Proof. Part (i) follows from [16, Theorem C.2]. For part (ii), let $A$ denote the Zalesskii subgroup of $G$ (see $[15$, p. 364] or [16, §1.5]). Then $A$ is a characteristic f.c. subgroup of $G$ of the form $A=D_{G}(H)$ for some finite-bynilpotent normal subgroup $H$ of $G$ and hence in particular, $A$ contains $F$. We consider the ideal $P \cap K[A]$ of $K[A]$. By Lemma 3.2, $P \cap K[A]$ can be expressed as $P \cap K[A]=P_{1} \cap P_{2} \cap \ldots \cap P_{n}$ with prime ideals $P_{i}$ of $K[A]$ which form an orbit under $\Gamma$. Therefore, the subgroups $P_{i}^{\dagger}$ of $\Gamma$ have only " finitely many conjugates under $\Gamma$ and

$$
\bigcap_{\gamma \in \Gamma}\left(P_{i}^{\dagger}\right)^{\gamma}=\left(\bigcap_{\gamma \in \Gamma} P_{i}^{\gamma}\right)^{\dagger}=(P \cap K[A])^{\dagger}=P^{\dagger} \cap A
$$

is a finite group. Since $\Gamma$ was assumed to be orbitally sound, we conclude that $P_{i}^{\dagger}$ is finite for all $i$, and hence $P_{i}$ is almost faithful. By [16, Theorem D], we obtain that

$$
P_{i}=\left(P_{i} \cap K\left[\mathbf{D}_{A}(\Gamma)\right]\right) K[A]=\left(P_{i} \cap K[F]\right) K[A]
$$

and hence

$$
P \cap K[A]=(P \cap K[F]) K[A]
$$

Therefore, we have to show that $P=(P \cap K[A]) K[G]$. By [15, Theorem 11.4.2], it suffices to check that $P \cap K[A]$ is annihilator free in $K[A]$, that is for any $\alpha \in K[A]$ and any infinite subgroup $B$ of $A, \alpha \cdot \omega(K[B]) \subset P \cap$ $K[A]$ implies that $\alpha \in P \cap K[A]$. But if $B$ is infinite, then $B \cap \mathscr{Z}(A)$ also is infinite and since $P_{i}^{\dagger}$ is finite, we may choose $x_{i} \in B \cap \mathscr{Z}(A), x_{i} \notin P_{i}^{\dagger}$. Then $x_{i}-1$ is nonzero and central modulo $P_{i}$ and hence is regular modulo $P_{i}$. Now if $\alpha \cdot \omega(K[B]) \subset P \cap K[A]$, then $\alpha\left(x_{i}-1\right) \in P_{i}$ and so $\alpha \in P_{i}$. Since this holds for all $i$, we have $\alpha \in \cap P_{i}=P \cap K[A]$ and we obtain that $P \cap K[A]$ is in fact annihilator free. The result follows.

We are now ready to prove the main result of this section.

Theorem 3.7. Let $\Gamma$ be polycyclic-by-finite, let $G \triangleleft \Gamma$ and let $P$ be $a \Gamma$-prime ideal of $K[G]$. Suppose the action of $\Gamma$ on $K[G] / P$ is almost fixed point free. Then there are countably many $\Gamma$-stable ideals $I_{1}, I_{2}, \ldots$ of $K[G]$ (not necessarily distinct from each other and from $K[G])$ with $I_{l} \supsetneq P$ and such that for any ideal $I$ of $K[G], \cap_{\gamma \in \Gamma} I^{\gamma} \supsetneqq P$ implies that $I \supset I_{l}$ for some $l$.

Proof. First observe that we may restrict ourselves to the case of prime ideals $I$. For, suppose we already have constructed a sequence $J_{1}, J_{2}, \ldots$ of $\Gamma$-stable ideals satisfying the requirements of the theorem for prime ideals $I$ containing $P$. If $I$ is any ideal of $K[G]$ containing $P$, then $I$ contains a finite product $P_{1} \cdot P_{2} \cdot \ldots \cdot P_{n}$ of primes with $P_{i} \supset I$. Clearly, if $\cap_{\gamma \in \Gamma} I^{\gamma} \neq P$, then $\cap_{\gamma \in \Gamma} P_{i}^{\gamma} \neq P$ for all $i$, and hence $P_{i}$ contains some of the ideals $J_{1}, J_{2}, \ldots$, say $P_{i} \supset J_{l(i)}$. But then $I \supset J_{l(1)} \cdot J_{l(2)} \cdot \ldots \cdot J_{l(n)}+P$, and the latter ideal is $\Gamma$-stable and strictly contains $P$, since $P$ is $\Gamma$-prime. Furthermore, the set of 
ideals of the form $J_{l(1)} \cdot J_{l(2)} \cdot \ldots \cdot J_{l(n)}+P$ clearly is a countable set and so the result follows in general.

Choose $\Gamma_{0}$ as in Theorem 3.6(i) and set $G_{0}=G \cap \Gamma_{0}$. Then, by Lemma 3.4, $P \cap K\left[G_{0}\right]$ can be written as a finite intersection $P \cap K\left[G_{0}\right]=\cap_{\gamma \in \Gamma} P_{0}^{\gamma}$, where $P_{0}$ is a $\Gamma_{0}$-prime of $K\left[G_{0}\right]$ such that the action of $\Gamma_{0}$ on $K\left[G_{0}\right] / P_{0}$ is almost fixed point free. We first establish the theorem for $\Gamma_{0}$ and $K\left[G_{0}\right] / P_{0}$. Since the orbitally sound property is inherited by homomorphic images, we may assume that $P_{0}$ is faithful, that is $P_{0}^{\dagger}=\langle 1\rangle$. Let $I_{0}$ be a prime ideal of $K\left[G_{0}\right]$ such that $\bar{I}_{0}=\cap_{\gamma \in \Gamma_{0}} I_{0}^{\gamma} \supset P_{0}$. Then $\bar{I}_{0}^{\dagger}$ is infinite, for otherwise application of Theorem 3.6(ii) would give that

$$
\bar{I}_{0}=\left(\bar{I}_{0} \cap K[F]\right) K\left[G_{0}\right] \text {, }
$$

where we have set $F=\mathbf{D}_{G_{0}}\left(\Gamma_{0}\right)$. But $\bar{I}_{0} \cap K[F] \supset P_{0} \cap K[F]$ and, by Lemma 3.5 applied to the $\Gamma_{0}$-prime ideal $P_{0} \cap K[F]$ of $K[F]$, we must in fact have equality. Thus we obtain that $\bar{I}_{0}=P_{0}$, a contradiction. Therefore, $\bar{I}_{0}^{\dagger}$ is infinite and the ideal $\left(\omega \bar{I}_{0}^{\dagger}\right) K\left[G_{0}\right]+P_{0}$ is strictly larger than $P_{0}$, since $P_{0}^{\dagger}=$ $\langle 1\rangle$. Furthermore, the ideal $\left(\omega \bar{I}_{0}^{\dagger}\right) K\left[G_{0}\right]+P_{0}$ is obviously $\Gamma_{0}$-stable and is a member of the countable set of ideals of the form $(\omega H) K\left[G_{0}\right]+P_{0}, H$ a normal subgroup of $G_{0}$. This yields the desired sequence $J_{1}, J_{2}, \ldots$ of ideals for $\Gamma_{0}$ and $K\left[G_{0}\right] / P_{0}$.

It remains to lift the result to $\Gamma$ and $K[G] / P$. So let $I$ be a prime ideal of $K[G]$ such that $\bar{I}=\bigcap_{\gamma \in \Gamma} I^{\gamma} \supset P$. Then, since $P$ is $\Gamma$-prime, $\bar{I}$ contains an element that is regular modulo $\stackrel{*}{P}$, by Lemma 1.1(i), and since $\left[G: G_{0}\right]<\infty$, we conclude from Lemma 2.2 that

$$
\bar{I} \cap K\left[G_{0}\right] \supsetneqq P \cap K\left[G_{0}\right]=\bigcap_{\gamma \in \Gamma} P_{0}^{\gamma} .
$$

Now $\bar{I}$ is a $\Gamma$-prime ideal of $K[G]$ and hence $\bar{I} \cap K\left[G_{0}\right]$ is a $\Gamma$-prime in $K\left[G_{0}\right]$, by Lemma 3.2. Therefore, Lemma 3.4 yields that

$$
\bar{I} \cap K\left[G_{0}\right]=\bigcap_{\gamma \in \Gamma} I_{0}^{\gamma}
$$

a finite intersection with $I_{0}$ a $\Gamma_{0}$-prime. After replacing $I_{0}$ by a $\Gamma$-conjugate if necessary, we have $I_{0} \supsetneqq P_{0}$. Therefore, by the result for $\Gamma_{0}$ and $K\left[G_{0}\right] / P_{0}$, the ideal $I_{0}$ contains some $J_{l}$ and hence

$$
I \cap K\left[G_{0}\right] \supset \bigcap_{\gamma \in \Gamma} I_{0}^{\gamma} \supset \bigcap_{\gamma \in \Gamma} J_{l}^{\gamma} \supset \bigcap_{\gamma \in \Gamma} P_{0}^{\gamma}=P \cap K\left[G_{0}\right]
$$

Here, the last inclusion is even strict. For otherwise, $J_{l}^{\gamma} \subset P_{0}$ for some $\gamma \in \Gamma$, since $\cap_{\gamma \in \Gamma} J_{l}^{\gamma}$ is a finite intersection of $\Gamma_{0}$-stable ideals and $P_{0}$ is $\Gamma_{0}$-prime. But then $J_{l} \supsetneqq J_{l}^{\gamma}$, which is impossible since $\Gamma$ acts as a finite permutation group. Therefore, $\cap_{\gamma \in \Gamma} J_{l}^{\gamma}$ and $P \cap K\left[G_{0}\right]$ are distinct and 


$$
I_{l}=\left(\bigcap_{\gamma \in \Gamma} J_{l}^{\gamma}\right) K[G]+P
$$

is a two-sided $\Gamma$-stable ideal of $K[G]$ strictly containing $P$ and contained in $I$. Since the ideals $I_{l}$ clearly form a countable set, the theorem is proved.

4. Topological methods. If not explicitly stated otherwise, the group $G$ is understood to be polycylic-by-finite throughout this section.

Given a ring $R$, the set $\operatorname{Spec}(R)$ of prime ideals of $R$ can be endowed with a topology, usually called the Jacobson topology, by declaring the subsets of the form

$$
\mathcal{V}(I)=\{P \in \operatorname{Spec}(R) \mid P \supset I\}, \quad I \text { an ideal of } R,
$$

to be closed. If $R=K[G]$ is the group algebra of a finitely generated nilpotent-by-finite group $G$, then the primitive ideals of $R$ turn out to be just those prime ideals $P$ of $R$ such that $\{P\}$ is a locally closed subset of the topological space $\operatorname{Spec}(R)$. Algebraically, the condition means that the intersection of all primes strictly containing $P$ is distinct from $P$ (cf. [13, Corollary 5.6]). Although this characterization of primitive ideals does not hold for general polycyclic groups, there is still some characterization of primitive ideals for these groups with a topological background. Unfortunately, this characterization only works for uncountable fields.

The crucial result we need is the following recent theorem of Farkas [4, Corollary 8].

THEOREM 4.1 (FARKAS). Let $K$ be an uncountable field and let $G$ be a polycyclic group. Then for any homomorphic image $R$ of $K[G]$ the $\operatorname{set} \operatorname{Priv}(R)$ of primitive ideals of $R$ is a Baire space (when considered as a subspace of $\operatorname{Spec}(R))$.

Recall that a Baire space is a topological space with the property that countable intersections of dense open subsets always are dense. We shall use Farkas' theorem in the following way.

Let $P$ be a prime ideal of $K[G]$ and let $R=K[G] / P$, which is a prime semiprimitive ring (cf. [9, Corollary 1.3]). Suppose Priv $(R)$ can be written as a union of countably many subsets $\mathscr{X}_{1}, \mathscr{X}_{2}, \ldots$ Then the conclusion of Farkas' theorem is that at least one of these has to be dense. For, if none of the $\mathscr{X}_{i}$ is dense, then for each $i$ the complement $\mathscr{V}_{i}$ of the closure $\overline{\mathscr{X}}_{i}$ is a nonempty open subset of $\operatorname{Priv}(R)$. But as a consequence of the fact that $R$ is prime and semiprimitive, any nonempty open subset of $\operatorname{Priv}(R)$ is dense and hence each $\mathcal{V}_{i}$ is dense. On the other hand, the intersection of the $\mathcal{V}_{i}$ is empty, which contradicts the Baire space property. Therefore, some $\mathfrak{X}_{i}$ has to be dense which, in algebraic terms, just means that $\cap_{Q \in \mathscr{X}_{i}} Q=(0)$.

In order to state the result we need some terminology. The ideal $P$ of $K[G]$ is said to have a separating set of ideals, if there exist countably many ideals 
$I_{1}, I_{2}, \ldots$ (not necessarily distinct from each other and from $K[G]$ ), all strictly containing $P$, with the property that any ideal strictly containing $P$ contains one of the ideals $I_{l}$. Observe that the existence of a separating set of ideals is trivial if $K$ is countable since a countable Noetherian ring has only countably many ideals. Hence for the following theorem we must have $K$ uncountable.

THEOREM 4.2. Let $K$ be an uncountable field and let $G$ be a polycyclic group. If $P$ is a prime ideal of $K[G]$, then the following are equivalent:

(i) $P$ is primitive;

(ii) the heart $\mathcal{H}(P)$ of $P$ is a finite algebraic extension of $K$;

(iii) $P$ has a separating set of ideals.

Proof. The equivalence of (i) and (ii) has been established in [13, Theorem 5.2], even under a less restrictive hypothesis on the field $K$. The implication (iii) $\Rightarrow$ (i) follows from Farkas' theorem (and is also in Farkas' paper [4, Theorem 1]). Namely, if $P$ is not primitive, then $\mathscr{X}=\operatorname{Priv}(K[G] / P)$ can be written as the union of the countably many subsets $\mathscr{X}_{l}=\{Q \in \mathcal{X} \mid Q \supset$ $\left.I_{l} / P\right\}$, where $I_{1}, I_{2}, \ldots$ is the separating ideal sequence for $P$. Consequently, some $\mathscr{X}_{i}$ has to be dense and so $\cap_{Q \in X_{i}} Q=(0)$. But $\cap_{Q \in X_{i}} Q$ contains the nonzero ideal $I_{i} / P$ of $K[G] / P$, a contradiction. Thus $P$ is in fact primitive.

Finally, (ii) $\Rightarrow$ (iii) follows from the results of $\S 3$. For, in the language of $\$ 3$, (ii) just says that the action of $G$ on $K[G] / P$ is almost fixed point free. So the existence of a separating set of ideals for $P$ follows from Theorem 3.7.

As a second application of the Baire space method we obtain the following result on the existence of "large" orbits of primitive ideals.

TheORem 4.3. Let $K$ be an uncountable field, let $P$ be a primitive ideal of $K[G]$ and let $N$ be a polycyclic normal subgroup of $G$. Then to any ideal $I$ of $K[N]$ such that $\cap_{g \in G} I^{g}=P \cap K[N]$ there exists a primitive ideal $Q$ of $K[N]$ with $Q \supset I$ and $\cap_{g \in G} Q^{g}=P \cap K[N]$.

Proof. First we reduce to the case $I$ prime. We have $I \supset P_{1} \cdot P_{2} \cdot \ldots \cdot P_{n}$ for suitable prime ideals $P_{i}$ of $K[N]$ with $P_{i} \supset I$. Therefore,

$$
P \cap K[N]=\bigcap_{g \in G} I^{g} \supset\left(\bigcap_{g \in G} P_{1}^{g}\right) \cdot\left(\bigcap_{g \in G} P_{2}^{g}\right) \cdot \ldots \cdot\left(\bigcap_{g \in G} P_{n}^{g}\right),
$$

since the latter ideal is obviously $G$-stable and is contained in $P_{1} \cdot P_{2}$ - .. $\cdot P_{n}$. Since $P \cap K[N]$ is $G$-prime, it follows that

$$
P \cap K[N] \supset \bigcap_{g \in G} P_{i}^{g}
$$

for some $i$. The other inclusion is clear, for $P \cap K[N] \subset I \subset P_{i}$. Hence we may assume that $I$ is prime.

By Corollary 3.3, the action of $G$ on $K[N] / P \cap K[N]$ is almost fixed point 
free, and Theorem 3.7 provides us with countably many $G$-stable ideals $I_{1}, I_{2}, \ldots$ of $K[N]$ with $I_{l} \supset P \cap K[N]$ for all $l$ and such that for any ideal $T$ of $K[N]$ with $T \supset P \cap K[N]$ and $T \nsupseteq I_{l}$ for all $l$, we have $\cap_{g \in G} T^{g}=P \cap$ $K[N]$.

Since $\cap_{g \in G} I^{g}=P \cap K[N]$, we clearly have $I \nsupseteq I_{l}$ for all $l$. Set $R=$ $K[G] / I$ and $J_{l}=\left(I_{l}+I\right) / I$ for $l=1,2, \ldots$ Then each $J_{l}$ is a nonzero ideal of the prime ring $R$. Set $\mathscr{X}=\operatorname{Priv}(R)$ and $\mathfrak{X}_{l}=\left\{T \in \mathscr{X} \mid T \supset J_{l}\right\}$. Since $\mathscr{X}$ is a Baire space, by Farkas' theorem, and since the $\mathcal{X}_{l}$ are countably many proper closed subsets of $\mathcal{X}$, it follows that $\mathfrak{X} \neq \cup_{l} \mathfrak{X}_{l}$. Choose $T \in$

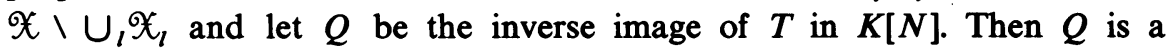
primitive ideal of $K[N]$ containing $I$, but not containing any of the ideals $I_{l}$. Therefore, by the fundamental property of these ideals, we must have $\cap_{g \in G} Q^{g}=P \cap K[N]$.

We close with a remark on the Jacobson property of $K[G]$ which answers in the positive a question by P. F. Smith in the special case of $K$ being uncountable. Recall that a ring $R$ is a Jacobson ring if and only if every prime ideal of $R$ is an intersection of primitive ideals.

Proposition 4.4. Let $K$ be an uncountable field and let $G$ be a polycyclic group. Then any faithful prime ideal of $K[G]$ can be written as an intersection of faithful primitive ideals.

Proof. Let $P$ be a faithful prime ideal of $K[G]$, that is

$$
P^{\dagger}=\{g \in G \mid 1-g \in P\}=\langle 1\rangle .
$$

To each element $g \in G, g \neq 1$, we associate an open subset $\mathfrak{X}_{g}$ of $\mathcal{X}=$ $\operatorname{Priv}(K[G] / P)$ by

$$
\mathfrak{X}_{g}=\{T \in \mathcal{X} \mid(1-g)+P \notin T\} .
$$

Since $P$ is faithful, the elements $(1-g)+P(g \neq 1)$ are all nonzero in $K[G] / P$ and hence the Jacobson property of $K[G]$ implies that the open subsets $\mathfrak{X}_{g}$ of $\mathcal{X}$ are nonempty and hence dense. Since they form a countable family of subsets of $\mathcal{X}$, the conclusion of Farkas' theorem is that the intersection

$$
\mathscr{D}=\bigcap_{g \in G \backslash\{1\}} \mathcal{X}_{g}
$$

is also dense, that is $\cap_{T \in \mathscr{D}} T=(0)$. But clearly, the inverse images in $K[G]$ of the ideals in $\mathscr{D}$ are faithful primitive ideals of $K[G]$, and their intersection equals $P$.

Added IN Proof. Farkas' theorem (Theorem 4.1) has meanwhile been extended to polycyclic-by-finite groups (M. Lorenz and D. S. Passman, Integrality and normalizing extensions of rings, J. Algebra, (to appear)). Thus the results in $\$ 4$ do in fact hold for polycyclic-by-finite groups. 


\section{REFERENCES}

1. W. Borho, P. Gabriel and R. Rentschler, Primideale in Einhüllenden auflösbarer Lie-Algebren, Springer, Berlin and New York, 1973.

2. N. Bourbaki, Éléments de mathématique, Fasc. XXX, Algèbre commutative, Actualitiés Sci. Indust., no. 1308, Hermann, Paris, 1964, Chap. 5.

3. J. Dixmier, Idéaux primitifs dans les algèbres enveloppantes, J. Algebra 48 (1977), 96-112.

4. D. Farkas, Baire category and Laurent extensions (to appear).

5. D. Farkas and D. S. Passman, Primitive noetherian group rings, Comm. Algebra 6 (1978), 301-315.

6. A. W. Goldie, The structure of noetherian rings, Lectures on Rings and Modules, Lecture Notes in Math., vol. 246, Springer, Berlin and New York, 1972.

7. A. W. Goldie and G. Michler, Ore extensions and polycyclic group rings, J. London Math. Soc. (2) 9 (1974), 337-345.

8. D. A. Jordan, Primitive skew Laurent polynomial rings, Glasgow Math. J. 19 (1978), 79-85.

9. M. Lorenz, Primitive ideals in group algebras of supersoluble groups, Math. Ann. 225 (1977), 115-122.

10. (1978), 285-294.

11. Completely prime primitive ideals in group algebras of finitely generated nilpotentby-finite groups, Comm. Algebra 6 (1978), 717-734.

12. _ The heart of prime ideals in Ore extensions, Manuscripta Math. (to appear).

13. M. Lorenz and D. S. Passman, Centers and prime ideals in group algebras of polycyclic-byfinite groups, J. Algebra 58 (1979), 355-386.

14. W. S. Martindale, Lie isomorphisms of prime rings, Trans. Amer. Math. Soc. 142 (1969), 437-455.

15. D. S. Passman, The algebraic structure of group rings, Wiley-Interscience, New York, 1977.

16. J. E. Roseblade, Prime ideals in group rings of polycyclic groups, Proc. London Math. Soc. (3) 36 (1978), 385-447.

Fachbereich Mathematik, Universitüt Essen, D-4300 Essen 1, Federal Rapublic of Germany (Current address)

Department of Mathematics, University of Wisconsin, Madison, Wisconsin 53706 